\title{
Manejo de paciente hipertenso em atendimento clínico farmacêutico: Um relato de
}

\author{
caso \\ Management of hypertensive patient in clinical pharmaceutical care: A case report \\ Manejo de pacientes hipertensos en atención farmacéutica clínica: Reporte de un caso
}

Recebido: 08/06/2021 | Revisado: 19/06/2021 | Aceito: 22/06/2021 | Publicado: 05/07/2021

\author{
Sthefane Silva Santos \\ ORCID: https://orcid.org/0000-0001-5985-7218 \\ Universidade Federal da Bahia, Brasil \\ E-mail: sthefaness@ufba.br \\ Amanda dos Santos Teles Cardoso \\ ORCID: https://orcid.org/0000-0002-9008-1021 \\ Universidade Federal da Bahia, Brasil \\ E-mail: amanda.teles@ufba.br \\ Ingrid Caroline da Silva Cerqueira \\ ORCID: https://orcid.org/0000-0002-0960-4156 \\ Universidade Federal da Bahia, Brasil \\ E-mail: ingridc@ufba.br \\ Renata Gonçalves Silva \\ ORCID: https://orcid.org/0000-0002-2909-4683 \\ Universidade Federal da Bahia, Brasil \\ E-mail: renatagsilva@ufba.br \\ Izabel Almeida Alves \\ ORCID: https://orcid.org/0000-0002-8935-6542 \\ Universidade Federal da Bahia, Brasil \\ E-mail: izabel.alves@ufba.br \\ Max Denisson Maurício Viana \\ ORCID: https://orcid.org/0000-0002-1650-4460 \\ Universidade Federal da Bahia, Brasil \\ E-mail: max.viana@ufba.br
}

\begin{abstract}
Resumo
Os serviços farmacêuticos são constituídos pelo acompanhamento farmacoterapêutico na identificação e resolução de problemas relacionados a medicamentos (PRMs) por meio de intervenções. O acompanhamento de pacientes hipertensos é fundamental, pois a hipertensão é considerada um dos principais fatores de risco que contribuem para a mortalidade de doenças cardiovasculares. No presente relato discorremos sobre os fatores de risco cardiovascular de paciente hipertensa e as intervenções farmacológicas e não farmacológicas realizadas para a resolução dos PRMs presentes. Trata-se de um estudo descritivo, do tipo relato de caso de paciente em consulta farmacêutica na Farmácia Universitária da Universidade Federal da Bahia, no período de outubro de 2019 a abril de 2020 (CEP n 2.578.262). Os questionários e intervenções foram baseadas no documento "Cuidado Farmacêutico na Atenção Básica: caderno 2”, do Ministério da Saúde. Relato de caso: Paciente do sexo feminino, 72 anos, diagnosticada com hipertensão há anos, apresenta comorbidades como dislipidemia, além de estilo de vida sedentário que, mutuamente, configuram-se como importantes fatores de risco cardiovascular. Praticava automedicação e apresentava diversos PRMs de adesão. Das intervenções farmacêuticas realizadas, incluíram: educação em saúde no que diz respeito ao uso racional de medicamentos (URM), ajustes para correção dos PRMs, incentivo à alimentação saudável e à prática de exercícios físicos. Frente à elaboração dos planos de intervenção farmacêutica, adotando terapias medicamentosas e não medicamentosas, possibilitou-se a redução dos níveis pressóricos, URM e a melhoria na qualidade de vida da paciente.
\end{abstract}

Palavras-chave: Doença cardiovascular; Cuidado farmacêutico; Educação em saúde.

\begin{abstract}
Pharmaceutical services consist of pharmacotherapeutic monitoring in the identification and resolution of drug-related problems (DRPs) through interventions. Monitoring hypertensive patients is essential, as hypertension is considered one of the main risk factors that contribute to mortality from cardiovascular diseases. In this report, we discuss cardiovascular risk factors in hypertensive patients and the pharmacological and non-pharmacological interventions performed to resolve the present DRPs. This is a descriptive study, of the case report type of a patient in a pharmaceutical consultation at the University Pharmacy of the Federal University of Bahia, from October 2019 to April $2020\left(\mathrm{CEP}^{\mathrm{o}}\right.$ 2.578.262). The questionnaires and interventions were based on the document "Pharmaceutical
\end{abstract}


Care in Primary Care: notebook 2", from the Ministry of Health. Case report: Female patient, 72 years old, diagnosed with hypertension for years, presents comorbidities such as dyslipidemia, in addition to style sedentary lifestyle that, mutually, configure themselves as important cardiovascular risk factors. She practiced self-medication and had several adherence DRPs. Of the pharmaceutical interventions performed, they included: health education regarding the rational use of medications (RUM), adjustments to correct the DRPs, encouragement of healthy eating and the practice of physical exercise. In view of the elaboration of pharmaceutical intervention plans, adopting drug and nondrug therapies, it was possible to reduce blood pressure levels, RUM and improve the patient's quality of life.

Keywords: Cardiovascular disease; Pharmaceutical care; Health education.

\section{Resumen}

Los servicios farmacéuticos consisten en el seguimiento farmacoterapéutico en la identificación y resolución de problemas relacionados con medicamentos (PRM) a través de intervenciones. El seguimiento de los pacientes hipertensos es fundamental, ya que la hipertensión se considera uno de los principales factores de riesgo que contribuyen a la mortalidad por enfermedades cardiovasculares. En este informe se discuten los factores de riesgo cardiovascular en pacientes hipertensos y las intervenciones farmacológicas y no farmacológicas realizadas para resolver los presentes PRM. Se trata de un estudio descriptivo, del tipo relato de caso de un paciente en consulta farmacéutica de la Farmacia Universitaria de la Universidad Federal de Bahía, de octubre de 2019 a abril de 2020 (CEP $\mathrm{n}^{\mathbf{0}}$ 2.578.262). Los cuestionarios e intervenciones se basaron en el documento "Atención Farmacéutica en Atención Primaria: cuaderno 2", del Ministerio de Salud. Caso clínico: Paciente de sexo femenino, 72 años, diagnosticada de hipertensión desde hace años, presenta comorbilidades como dislipidemia, además a estilizar un estilo de vida sedentario que, mutuamente, se configuran como importantes factores de riesgo cardiovascular. Practicó la automedicación y tuvo varios PRM de adherencia. De las intervenciones farmacéuticas realizadas, estas incluyeron: educación para la salud sobre el uso racional de medicamentos (URM), ajustes para corregir los PRM, fomento de la alimentación saludable y la práctica de ejercicio físico. Ante la elaboración de planes de intervención farmacéutica, adoptando terapias farmacológicas y no farmacológicas, se logró reducir los niveles de presión arterial, URM y mejorar la calidad de vida del paciente.

Palabras clave: Enfermedad cardiovascular; Cuidado farmacéutico; Educación para la salud.

\section{Introdução}

A realização dos serviços farmacêuticos é fundamental para a promoção da qualidade de vida e para o alcance de resultados concretos em saúde. Tais serviços se referem ao conjunto de ações efetuadas sistematicamente sendo constituídos, por exemplo, pelo acompanhamento farmacoterapêutico na análise e identificação de PRMs, bem como na realização de intervenções devidamente documentadas visando resolvê-los ou preveni-los, além da revisão da farmacoterapia (Conselho Federal de Farmácia, 2013; CFF 2016).

Um estudo nacional sobre as concepções de profissionais de saúde da Atenção Básica a respeito da Assistência Farmacêutica apresentou múltiplas compreensões que revelaram uma mudança de visão da AF centrada na distribuição de medicamentos para o enfoque no paciente e suas necessidades em saúde (Costa et al., 2017). Esse novo paradigma em que a Atenção Farmacêutica é fundamental para o acesso e o uso racional de medicamentos (URM) insere o farmacêutico na equipe multiprofissional de saúde apoiando os demais profissionais e o paciente na busca de melhores resultados com a farmacoterapia.

De acordo com a Organização Mundial de Saúde (OMS), nos últimos vinte anos, as doenças cardiovasculares (DCV) se configuraram como as principais causas de morte no mundo. No que se refere às doenças cardíacas, como a hipertensão arterial sistêmica (HAS), o número de mortes aumentou de mais de 2 milhões desde o ano 2000 para quase 9 milhões em 2019 (OPAS, 2020). A HAS é uma condição clínica multifatorial, crônica não transmissível, caracterizada pela pressão arterial sistólica (PAS) $\geq 140 \mathrm{mmHg}$ e pressão arterial diastólica (PAD) $\geq 90 \mathrm{mmHg}$ como valores persistentes (Barroso et al., 2020).

A causa da HAS é muito discutida no âmbito científico. Contudo, aponta-se uma combinação de fatores de risco com potenciais associações, a saber: doenças pré-existentes, hábitos de vida inadequados ou ainda variáveis individuais, aumentando consideravelmente o risco cardiovascular. Assim, é evidenciada a necessidade de compreendê-los para facilitar a implementação de medidas preventivas e terapêuticas apropriadas para hipertensos (Coelho, 2006). Dentre os principais fatores 
de risco cardiovascular, destacam-se: idade, sexo, fatores socioeconômicos, excesso de peso, consumo excessivo de sal, hábitos etilista e tabagista, sedentarismo, além das dislipidemias, história de DCV prematura em parentes de $1^{\circ}$ grau e diabetes mellitus (Barroso et al., 2020).

A estratificação do risco cardiovascular está relacionada à probabilidade de morte por um evento cardiovascular, como infarto agudo do miocárdio, e sua utilização está associada a melhor qualidade da atenção e maior eficácia no uso dos recursos de saúde (Sousa et al., 2016). Assim, conhecer a capacidade de autocuidado, os hábitos de saúde, os medicamentos em uso e as complicações secundárias às doenças de base de cada paciente, permite uma estratificação individualizada e o reconhecimento do nível de atenção e cuidado necessários para assisti-los (Coppini, 2017).

Tendo em vista os fatores de risco para mortalidade de DCVs, é fundamental o exercício do farmacêutico no processo de cuidado e manejo do tratamento de pacientes com HAS, no sentido do estímulo e promoção do autogerenciamento e autocuidado em saúde em todas as esferas e, consequentemente, na redução da PA a níveis aceitáveis (Conselho Regional de Farmácia - São Paulo, 2010), além de prevenir a progressão de DCVs através de intervenções farmacológicas e/ou nãofarmacológicas e monitoramento da farmacoterapia.

Baseado na importância do reconhecimento e consolidação da atuação do farmacêutico clínico no que tange à estratificação do risco cardiovascular em pacientes hipertensos, o presente estudo teve como objetivo discutir as intervenções farmacêuticas realizadas para resolução de problemas relacionados a medicamentos (PRMs) e prevenção da progressão de doenças crônicas não transmissíveis (DCNTs), como a HAS, a partir da análise de um caso clínico de paciente idosa e cardiopata assistida pela Farmácia Universitária da Universidade Federal da Bahia (FU-UFBA).

\section{Metodologia}

Trata-se de um estudo qualitativo, descritivo, do tipo relato de caso (Pereira et al., 2018), no qual se avaliou a estratificação do risco cardiovascular em paciente hipertensa atendida em consulta farmacêutica na FU-UFBA, sob aprovação do Comitê de Ética em Pesquisa (CEP) n n 2.578.262. O período de acompanhamento compreendeu os meses de outubro de 2019 a abril de 2020. As caracterizações e classificações dos PRMs e intervenções farmacêuticas foram baseadas no documento "Cuidado Farmacêutico na Atenção Básica: caderno 2", do Ministério da Saúde (2014).

A FU-UFBA, situada na Faculdade de Farmácia da UFBA, em Salvador-Bahia, compreende um projeto de extensão que realiza ações de educação em saúde e acompanhamento farmacoterapêutico de portadores de DCNT. A seleção dos pacientes é realizada através da manifestação voluntária do interesse do paciente após oferta do serviço farmacêutico durante sala de espera realizada no Laboratório de Análises Clínicas e Toxicológicas da Faculdade de Farmácia (LACTFAR-UFBA). Os pacientes cadastrados, devidamente orientados e que concordam com a proposta, assinam o Termo de Consentimento Livre e Esclarecido (TCLE) e participam de consultas farmacêuticas que visam melhorar os resultados com a farmacoterapia e a qualidade de vida do participante. As consultas são guiadas por farmacêuticos institucionais e acompanhadas pelos discentes participantes do projeto. O registro clínico das consultas farmacêuticas é realizado em formulário próprio do projeto, via software Microsoft Excel, com base no método clínico SOAP (subjetivo, objetivo, avaliação e plano) (Hurley, 1998) (Figura 1). 
Figura 1. Fases que compreendem o atendimento farmacêutico na Farmácia Universitária da UFBA.

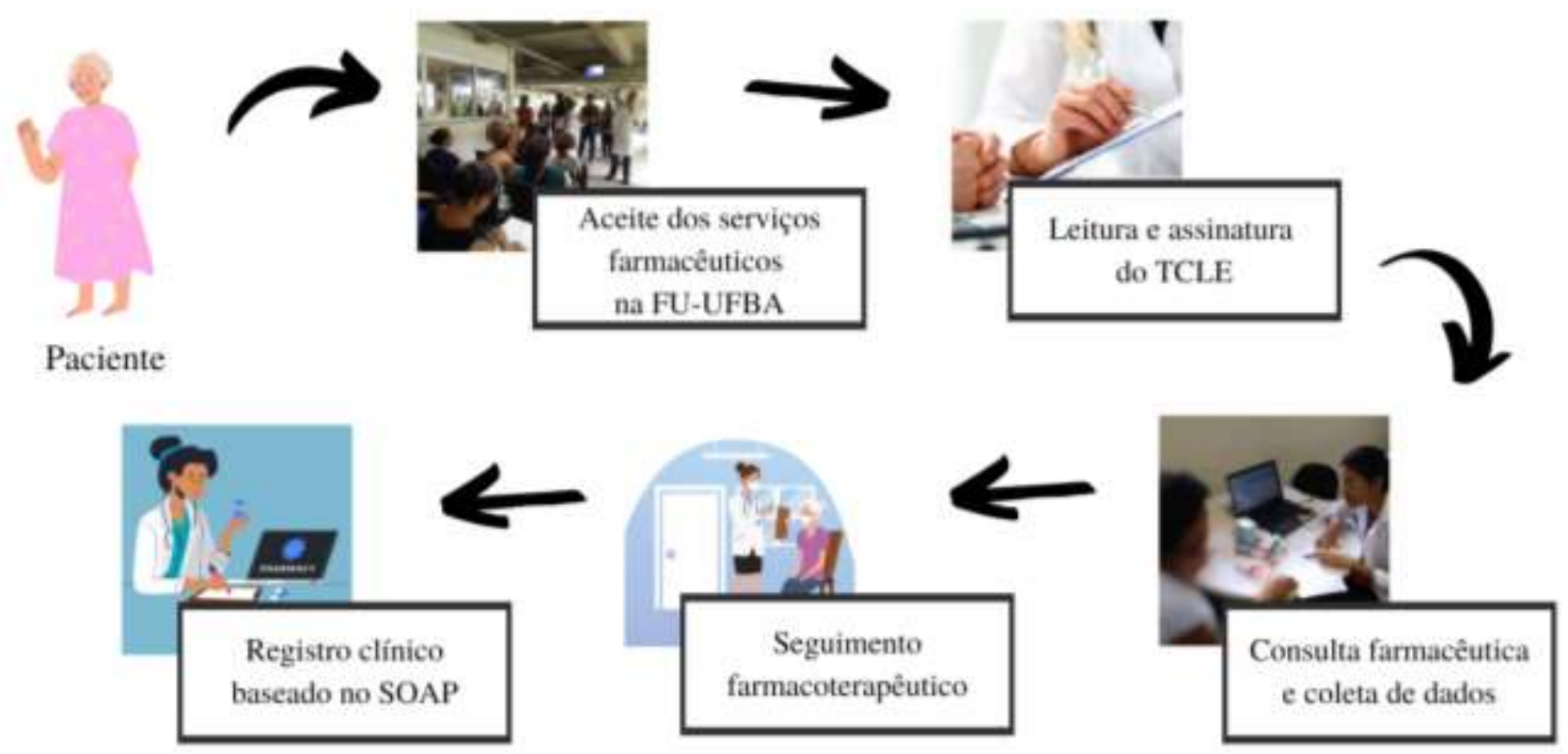

Fonte: Autores (2021).

\section{Relato de Caso}

Paciente do sexo feminino, 72 anos, 1,53 m de altura, $67 \mathrm{~kg}$, IMC 28,62, diagnosticada com HAS, dislipidemia e osteoporose. Durante a consulta farmacêutica, paciente relatou histórico familiar prematuro de DCV em parente de primeiro grau, pois seu irmão sofreu infarto agudo do miocárdio aos 28 anos. Em relação aos fatores de risco cardiovascular, afirmou estar sedentária, negou práticas tabagista e etilista e possuía dieta escassa em ácidos graxos poliinsaturados e fibras. Sobre o uso de medicamentos, relatou fazer uso por automedicação da associação de citrato de orfenadrina $35 \mathrm{mg} / \mathrm{mL}+$ dipirona monohidratada $300 \mathrm{mg} / \mathrm{mL}$ + cafeína $50 \mathrm{mg} / \mathrm{mL}$ para dor, do medicamento homeopático composto por Agnus castus $6,25 \mu \mathrm{L}$ + Conium maculatum 6,25 $\mu \mathrm{L}+$ Nuphar luteum $6,25 \mu \mathrm{L}+$ Onosmodium virginianum 6,25 $\mu \mathrm{L}$, indicado pela vizinha como "vitamina", o qual a paciente usou por um curto período relatando indiferença na eficácia terapêutica e do cloridrato de loperamida $2 \mathrm{mg}$ para diarreia ocasional, associada a quadros emocionais. Entre os medicamentos prescritos constavam: um complexo vitamínico, que a paciente associava à polifagia e aumento da disposição, além dos anti-hipertensivos: losartana 50 mg e hidroclorotiazida $25 \mathrm{mg}$ (ambos prescritos para uso por uma vez ao dia, pela manhã), o alendronato de sódio 70 mg (uma vez por semana) para osteoporose e o ácido acetilsalicílico $100 \mathrm{mg}$ (uma vez por dia após almoço), como antiagregante plaquetário. A respeito da utilização dos medicamentos prescritos, a paciente relatou não utilizar regularmente por desconhecimento da necessidade de uso diário.

Na primeira consulta, a PA se encontrava em 160 x $80 \mathrm{mmHg}$. Paciente apresentou resultado de exames laboratoriais recentes com valores de colesterol total de $265 \mathrm{mg} / \mathrm{dL}$ e lipoproteína de baixa densidade (LDL-c) de $176 \mathrm{mg} / \mathrm{dL}$. Perante a avaliação do caso e por meio da Calculadora para Estratificação do Risco Cardiovascular da Sociedade Brasileira de Cardiologia (SBC, 2020), a paciente foi considerada com alto risco cardiovascular. Além disso, foram identificados: PRM de adesão, pois foi constatada descontinuação dos anti-hipertensivos e automedicação indevida; e interações medicamentosas relevantes entre a associação de orfenadrina e cafeína com os anti-hipertensivos. Ainda, estava em posse diversos medicamentos vencidos, cartelas cortadas e sem data de validade.

Diante disso, as intervenções farmacêuticas consistiram em alterações e sugestões na farmacoterapia, como a suspensão dos medicamentos em uso por automedicação, no aconselhamento da paciente sobre a importância e aumento da 
adesão ao tratamento medicamentoso, e dos riscos da automedicação, acesso e armazenamento dos medicamentos. Ademais, foram realizados encaminhamentos a outros profissionais para avaliação da dor e foi recomendado o automonitoramento dos níveis pressóricos a fim de avaliar a efetividade do tratamento anti-hipertensivo após adesão à farmacoterapia. Tais ações, atreladas às intervenções não-farmacológicas, como o incentivo ao consumo de alimentos saudáveis e à prática de exercícios físicos, contribuíram para que ao retorno, quatro meses depois, a redução dos níveis pressóricos fosse constatada, com valor 148 x $85 \mathrm{mmHg}$ na consulta, e melhor seguimento da farmacoterapia pela paciente.

\section{Discussão}

No caso clínico deste estudo, a atuação do farmacêutico junto à paciente resultou em intervenções farmacêuticas importantes, especialmente não farmacológicas, que contribuíram para a melhoria da qualidade de vida da paciente. No geral, foram recomendadas práticas que minimizariam o sedentarismo no intuito de reduzir o impacto dos fatores de risco e orientações no sentido de uma alimentação mais saudável. Com idade avançada, hipertensão, dislipidemias, sedentarismo e má alimentação, a paciente se enquadra em mais de três fatores de risco, caracterizando-a como alto risco cardiovascular (Barroso et al., 2020). Características próprias ao envelhecimento podem contribuir para a presença destes fatores de risco, devido às alterações estruturais e funcionais na vasculatura arterial além de outros mecanismos subjacentes, como a rigidez arterial e redução das funções renais. O conjunto desses fatores associados ao histórico familiar prematuro de DCV resulta no aumento do risco cardiovascular (Oliveros et al., 2020).

Para pacientes com altos níveis dislipidêmicos, a recomendação de uma boa alimentação pode influenciar na redução do lipidograma. Mais especificamente dieta rica em proteínas de origem vegetal e ácidos graxos insaturados, em especial, ácidos graxos poliinsaturados ômega-3, provenientes de fontes marinhas como peixes sardinha, salmão e atum; e em sementes, sendo a linhaça a fonte mais abundante (Stefanello, Pasqualotti \& Pichler, 2019; Trautwein \& Mckay, 2020). Em vista da correlação entre níveis elevados do LDL-c, infarto agudo do miocárdio e morte cardiovascular (Berman \& Blankstein, 2018), por meio da Diretriz Brasileira de Dislipidemias e Prevenção da Aterosclerose, a SBC define como meta primária a redução do LDL-c para pacientes com alto risco cardiovascular para menores que $70 \mathrm{mg} / \mathrm{dL}$ (SBC, 2017).

Ainda, o consumo excessivo de sal relatado pela paciente é considerado o principal fator de risco dietético para as DCNTs, devido à relação com HAS, recomendando-se uma redução para o controle da PA. Apesar do conhecimento dos efeitos nocivos, pouco se sabe a respeito dos níveis adequados de ingestão. Em estudo recente sobre o consumo de sal na população adulta brasileira, constatou-se alta frequência em todo o país (Mill et al., 2021). O sistema renina-angiotensinaaldosterona, responsável pela regulação da PA, pelo balanço hídrico e de íons de sódio, é influenciado pela ingestão e, consequentemente, sua ativação inapropriada tem como consequência a HAS (Martins, 2016; Gonsalez et al, 2018).

Ademais, a inclusão de uma postura mais ativa e menos sedentária da paciente, e maior envolvimento de atividades físicas incluídas na rotina conforme orientado pelo farmacêutico, pode ter contribuído para redução nos níveis pressóricos, uma vez que a prática insuficiente e o sedentarismo, tem como consequências o sobrepeso, obesidade, elevação dos triglicerídeos e redução do HDL-c culminando na elevação da PA (González, Fuentes \& Márquez, 2017).

Na revisão da farmacoterapia utilizada pela paciente, foram constatadas interações medicamentosas ocasionadas pela politerapia. A maior ocorrência de doenças e politerapia por idosos pode se relacionar com a maior incidência da automedicação e de interações medicamentosas nessa faixa etária. O uso simultâneo de múltiplos medicamentos é uma prática comum e pode gerar um evento clínico caracterizado pelo aumento ou diminuição do efeito terapêutico ou tóxico de fármacos (Beserra et al., 2019; Rill, 2016; Hoefler, 2020). 
No caso, foi observada uma possível interação entre os anti-hipertensivos, losartana e hidroclorotiazida, e o antiinflamatório não-esteroidal (AINE), ácido acetilsalicílico, utilizado no tratamento como agente antiplaquetário. O mecanismo de tal interação está relacionado com o fato de o AINE causar inibição da enzima ciclooxigenase, o que gera uma redução sistêmica e renal da síntese de prostaglandinas, promovendo a diminuição da excreção renal de sódio e, consequentemente, diminui a eficácia dos medicamentos anti-hipertensivos (Nascimento \& Pigoso, 2013). Outra possível interação encontrada ocorreu entre o alendronato de sódio e o AINE utilizado, uma vez que ambos os fármacos podem provocar alto grau erosivo no estômago, elevando o risco de gastrite e úlceras gastrointestinais quando utilizados concomitantemente (DRUGS, 2021).

Referente à automedicação, a paciente estava em uso de medicamento composto por cafeína que pode contribuir para oscilações na PA (Benjamin et al., 2021). Também, de medicamento homeopático ativador sexual por indicação da vizinha como "vitaminas", além de cloridrato de loperamida na ocorrência de diarreias, que oferece um risco à saúde relativo à ação constipante intensa podendo propiciar um ambiente intestinal favorável para a proliferação de bactérias patogênicas devido ao atraso na eliminação (Araújo, 2014). A análise da automedicação, além de alertar o farmacêutico quanto à exposição aos riscos à saúde, pode ser interpretado como um gatilho que revela a existência de um problema de saúde não tratado relacionado com seu histórico familiar e psicológico, necessitando do envolvimento de outros profissionais de saúde para melhoria da qualidade de vida da paciente, como o psicólogo.

A paciente também apresentou PRMs de adesão à farmacoterapia anti-hipertensiva prescrita, reconhecidos pela descontinuação indevida e, consequentemente, a descompensação da PA. Embora os anti-hipertensivos utilizados sejam eficazes com perfil de reações adversas discreto, é comum a baixa adesão quando se trata de DCNT, uma vez que o caráter crônico da doença requer o uso contínuo do medicamento, o que exige mudanças no hábito de vida. Em geral, a não adesão a medicamentos pode estar relacionada com obstáculos específicos dos pacientes, como: depressão, comorbidades, baixo nível de alfabetização em saúde, custo e preocupações com o medicamento, esquecimento e falta de motivação para o autocuidado (Peacock \& Krousel-wood, 2018).

Outro PRM de adesão à farmacoterapia foi relacionado ao tratamento da osteoporose em que a paciente não cumpria corretamente as recomendações pós-administração do alendronato de sódio por falta de orientação. O PRM condicionava a paciente ao risco de desenvolvimento de esofagite pela característica do fármaco de irritação da mucosa. Assim, as orientações quanto ao uso correto do medicamento realizadas pelo farmacêutico, como a ingestão em jejum, com bastante água, e manterse em pé ou sentada por cerca de 30 minutos após administração do medicamento, foram fundamentais para a promoção do seu uso racional.

Ainda, a paciente apresentou na consulta diversos medicamentos vencidos, cartelas cortadas e sem data de validade. É importante frisar que os medicamentos devem ser consumidos no período e dosagens prescritas corretamente, e devem ser descartados em postos de coleta (CRF-PR, 2018). A confecção de envelopes individuais contendo as cartelas dos medicamentos, bem como horários de uso, foi uma importante estratégia de intervenção para reduzir os riscos do uso irracional de medicamentos e PRMs de adesão; além de orientações quanto ao descarte correto de medicamentos.

As ações do farmacêutico junto à paciente, como a suspensão da automedicação e as orientações quanto ao uso correto dos medicamentos para hipertensão e osteoporose, apoiam o autocuidado, além de melhorar a gestão autônoma dos medicamentos ampliando a compreensão sobre seu tratamento. O Quadro 1 sistematiza as intervenções farmacêuticas realizadas para o caso estudado. 
Quadro 1. Levantamento de intervenções farmacológicas realizadas no caso.

\begin{tabular}{|c|c|c|c|c|c|}
\hline $\begin{array}{c}\text { Problema } \\
\text { de saúde }\end{array}$ & Medicamentos & Posologia & Frequência & PRM & Intervenções* \\
\hline \multirow{2}{*}{ Hipertensão } & Losartana & $50 \mathrm{mg}$ & \multirow{2}{*}{$\begin{array}{l}\text { Uma vez pela } \\
\text { manhã }\end{array}$} & \multirow{2}{*}{$\begin{array}{l}\text { De adesão: Descontinuação } \\
\text { indevida. }\end{array}$} & \multirow{2}{*}{$\begin{array}{c}\text { Orientações quanto aos horários } \\
\text { do uso dos medicamentos e da } \\
\text { importância de utilizá-los } \\
\text { diariamente. }\end{array}$} \\
\hline & Hidroclorotiazida & $25 \mathrm{mg}$ & & & \\
\hline Osteoporose & Alendronato de sódio & $70 \mathrm{mg}$ & $\begin{array}{l}\text { Uma vez por } \\
\text { semana }\end{array}$ & $\begin{array}{l}\text { De adesão / administração: } \\
\text { Técnica de administração } \\
\text { do paciente incorreta. }\end{array}$ & $\begin{array}{c}\text { Orientações acerca da } \\
\text { administração para evitar } \\
\text { irritação gastrointestinal (efeito } \\
\text { adverso grave). }\end{array}$ \\
\hline $\begin{array}{l}\text { Dores } \\
\text { proveniente } \\
\text { s da } \\
\text { osteoporose }\end{array}$ & $\begin{array}{l}\text { Orfenadrina, Dipirona } \\
\text { Monoidratada e } \\
\text { Cafeína, em } \\
\text { associação }\end{array}$ & $\begin{array}{c}35 \mathrm{mg} / \mathrm{mL}, \\
300 \mathrm{~g} / \mathrm{mL} \mathrm{e} \\
50 \mathrm{mg} / \mathrm{mL}\end{array}$ & $\begin{array}{l}\text { Quando sente } \\
\text { dores }\end{array}$ & $\begin{array}{l}\text { De adesão: Automedicação } \\
\text { indevida. }\end{array}$ & $\begin{array}{c}\text { Orientações quanto à suspensão } \\
\text { do medicamento devido às } \\
\text { contribuições para oscilações na } \\
\text { PA. }\end{array}$ \\
\hline Diarreia & $\begin{array}{l}\text { Cloridrato de } \\
\text { loperamida }\end{array}$ & $2 \mathrm{mg}$ & Regularmente & $\begin{array}{l}\text { De adesão: Automedicação } \\
\text { indevida. }\end{array}$ & $\begin{array}{c}\text { Orientações quanto à suspensão } \\
\text { do medicamento devido às } \\
\text { contribuições para proliferação } \\
\text { de bactérias patogênicas no } \\
\text { intestino. }\end{array}$ \\
\hline
\end{tabular}

Fonte: Autores (2021).

Diante disso, o exercício do profissional farmacêutico no acompanhamento de pacientes portadores de DCNTs é importante na análise da terapia medicamentosa, suas possíveis interações e reações adversas e no desenvolvimento de estratégias educacionais quanto ao uso de medicamentos (Oliveira, Filipin \& Giardini, 2015). Ações farmacêuticas semelhantes às realizadas no caso são encontradas na literatura e demonstram a redução da PAS de $150 \mathrm{mmHg}$ para 120 mmHg devido ao acompanhamento farmacoterapêutico que permitiu a resolução de PRMs de adesão, educação em saúde e boa aceitabilidade por parte dos pacientes, resultando na melhoria do quadro clínico e qualidade de vida (Campos et al., 2020; Aires \& Marchiorato, 2010). Tais contribuições são evidenciadas em estudo do impacto da consulta farmacêutica em pacientes polimedicados com alto risco cardiovascular, no qual foi demonstrado o controle das comorbidades dos pacientes com diferença significativa observada em curto prazo, além da melhor adesão ao tratamento, garantindo desfechos positivos na saúde do paciente (Reis et al., 2018). Vale ressaltar que pacientes portadores de DCNTs são acompanhados continuamente, os atendimentos ocorrem de maneira processual, assim como a evolução dos pacientes é facilitada com a construção da relação com o farmacêutico. Desta forma, as medidas de controle da automedicação, identificação de interações medicamentosas e intervenções não-farmacológicas foram essenciais para os resultados alcançados, conforme demonstrado na Figura 3. 
Figura 3. Principais fatores de risco cardiovascular presentes na paciente e sua evolução antes e depois das intervenções farmacêuticas.

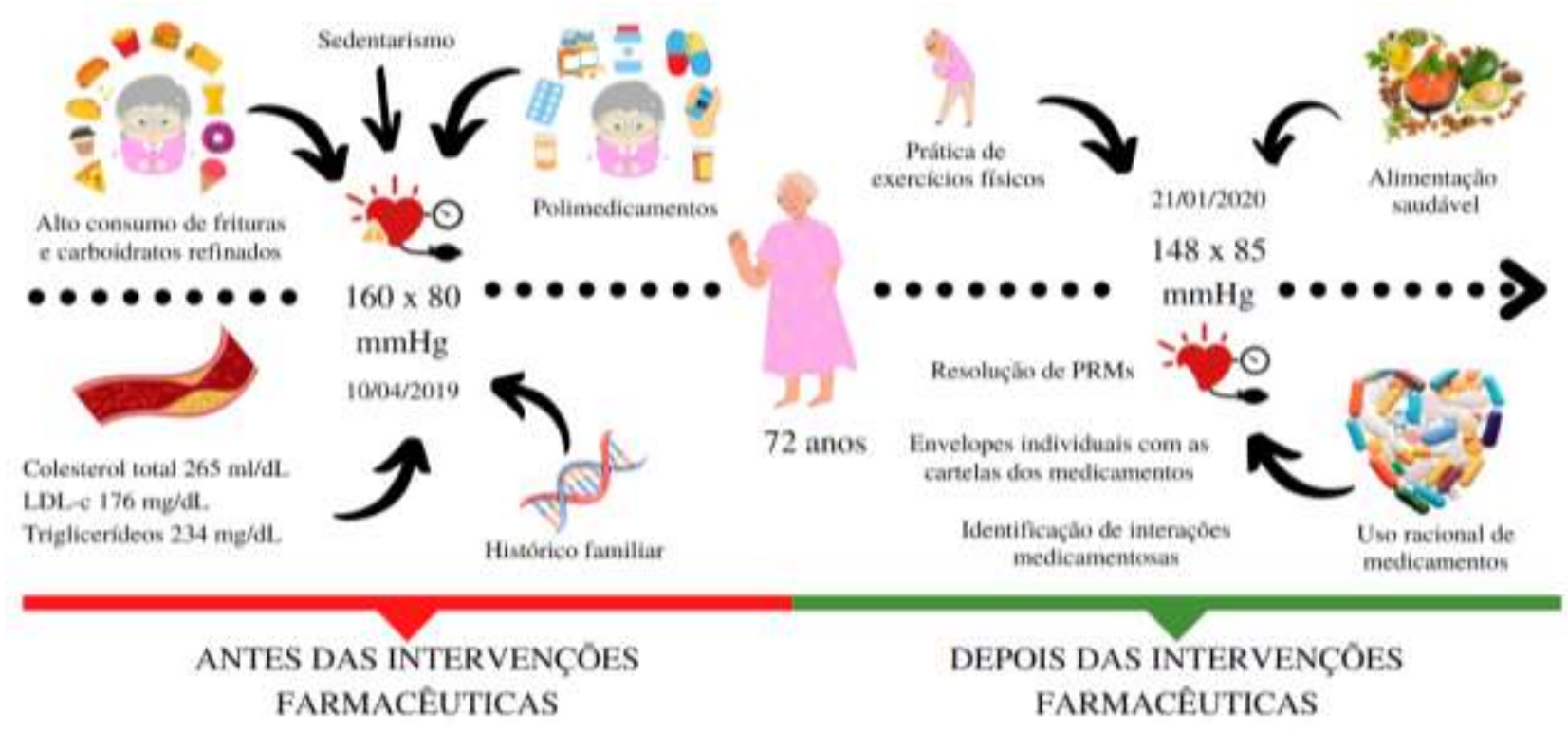

Fonte: Autores (2021).

\section{Conclusão}

A atuação do farmacêutico na identificação de PRMs, interações medicamentosas e estratificação do risco cardiovascular foi fundamental para o planejamento do plano de cuidado da paciente hipertensa, visto que estão relacionados à adesão dos medicamentos e automedicação. Estes parâmetros, quando associados à posse de cartelas de medicamentos cortadas, sem validade e com o prazo de validade expirado, maus hábitos alimentares bem como estilo de vida sedentário, podem interferir na evolução do tratamento.

O seguimento farmacoterapêutico foi realizado visando a adoção de terapias farmacológicas e não farmacológicas, mudanças no estilo de vida, a análise, identificação e a resolução de PRMs, promoção do URM e a educação em saúde mediante à orientação sobre a importância da administração e horários corretos no uso de medicamentos, bem como do descarte. A adesão da paciente às intervenções propostas foi fundamental para alcançar bons resultados e melhora geral do quadro clínico. Nesse sentido, prospera-se um maior acompanhamento dessa paciente, assim como de outros pacientes cardiopatas assistidos pela FU-UFBA, visando melhores desfechos clínicos.

\section{Agradecimentos}

Agradecemos a Universidade Federal da Bahia e a toda equipe da Farmácia Universitária da UFBA.

\section{Referências}

Aires, C. C. N. F \& Marchiorato, L. (2010). Acompanhamento farmacoterapêutico a hipertensos e diabéticos na Unidade de Saúde Tereza Barbosa análise de caso. Revista Brasileira de Farmácia Hospitalar e Serviços de Saúde, 1 (1), 26-31.

Araújo, C. M. A. (2014). Tratamento da diarreia aguda (Dissertação). Universidade Fernando Pessoa, Faculdade de Ciências da Saúde: Porto.

Aziz, J. L. (2014). Sedentarismo e hipertensão arterial. Revista Brasileira de Hipertensão, 21 (2), 75-82.

Barroso, W. K. S., et al. (2020). Diretrizes Brasileiras de Hipertensão Arterial. Arquivo Brasileiro de Cardiologia.

Benjamin, C. J. R. et al. (2021). Ação da Cafeína no Sistema Nervoso Central e na Variabilidade da Frequência Cardíaca. Id on Line Revista Multidisciplinar e de Psicologia, 15(54), 405-409. 
Berman, A. N. \& Blankstein, R. (2019). Optimizing Dyslipidemia Management for the Prevention of Cardiovascular Disease: a Focus on Risk Assessment and Therapeutic Options. Current Cardiology Reports, 21 (9), 1-10.

Bertoldi, G. (2012). A importância do educador físico na avaliação e prescrição de exercícios físicos para o controle da diabetes e da hipertensão arterial sistêmica (Especialização em Gestão de Organização Pública em Saúde Conclusão de Curso). Universidade Federal de Santa Maria, Tio Hugo, RS

Beserra, F. L. P. R., Borba, V. F. C., Torres, J. E. G., Silva, S. N. D. \& Macedo, M. A. C. S. (2019). Automedicação em idosos: medidas de prevenção e controle. Revista Contexto \& Saúde, 19 (37), 149-155.

Campos, L. S. (2020). A prática da atenção farmacêutica no acompanhamento farmacoterapêutico de idosos diabéticos e hipertensos: relato de caso. Brazilian Journal of Health Review, 3 (2), 2287-2296.

Coelho, A. M. (2006). Estratificação de risco cardiovascular em doentes hipertensos de uma lista de utentes. Revista Portuguesa de Medicina Geral e Familiar, 22 (1), 41-48

Coppini, L. A. Jr. (2017). Estratificação de risco e determinação de ações em atenção básica para hipertensos e diabéticos [Trabalho de Conclusão de Curso]. Porto Alegre: Universidade Federal de Ciências Da Saúde de Porto Alegre.

Costa, E. A. et al. (2017). Concepções de assistência farmacêutica na atenção primária à saúde, Brasil. Revista de Saúde Pública, 51(Supl. 2)5s

De Farmácia do Estado de São Paulo, C. R. (2010). Fascículo IV - Manejo do Tratamento de Pacientes com Hipertensão. Projeto Farmácia Estabelecimento de Saúde: Conselho Regional de Farmácia do Estado de São Paulo.

De Farmácia do Paraná, C. R. (2018). Descarte de Medicamentos: Conselho Regional de Farmácia do Estado do Paraná. https://www.crfpr.org.br/pagina/visualizar/291

De Farmácia, C. F. (2013). Resolução CFF n 585/2013. Regulamenta as atribuições clínicas do farmacêutico e dá outras providências: Conselho Federal da Farmácia.

De Farmácia, C. F. (2016). Serviços farmacêuticos diretamente destinados ao paciente, à família e à comunidade: contextualização e arcabouço conceitual: Conselho Federal de Farmácia.

Drugs.com (2021). Drug Interaction Report [Internet]. https://www.drugs.com/interactions-check.php?drug_list=116-0,243-0

Gonsalez, S. R., Ferrão, F. M., Souza, A. M., Lowe, J. \& Morcillo, L. S. L. (2018). Atividade inadequada do sistema renina-angiotensina-aldosterona local durante período de alta ingestão de sal: impacto sobre o eixo cardiorrenal. Brazilian Journal of Nephrology, 40 (2), 170-178.

González, K., Fuentes, J \& Márquez, J. L. (2017). Physical Inactivity, Sedentary Behavior and Chronic Diseases. Korean Journal of family medicine, 38 (3), $111-115$.

Hoefler, R. (2012). Interações medicamentosas. Secretaria de Ciência, Tecnologia e Insumos Estratégicos/MS - FTN.

Hurley, S. C. (1998). A Method of Documenting Pharmaceutical Care Utilizing Pharmaceutical Diagnosis. American Journal of Pharmaceutical Education, $62,119-127$.

Kruk, P. J. \& Nowicki, M. (2018). Effect of the physical activity program on the treatment of resistant hypertension in primary care. Primary health care research \& development, 19 (6), 575-583.

Lima, M. R. S. et al. (2019). A visão dos agentes comunitários de saúde sobre a importância do nutricionista na atenção básica. Brazilian Journal of health review, 2(6), 6041-6049.

Martins, D. S. (2016). Relação entre hipertensão e o consumo de sal: uma revisão (Monografia). Universidade Federal de Campina Grande, Cuité, PB.

Mill, J. G. et al. (2021). Fatores associados ao consumo de sal na população adulta brasileira: Pesquisa Nacional de Saúde. Ciência \& Saúde Coletiva, 26 (2), $555-567$.

Nascimento, D. M. \& Pigoso, A. A. (2013). Interações medicamentosas entre antihipertensivos e anti-inflamatórios não esteroidais. Revista Científica da FHO|UNIARARAS, 1 (1), 14-17.

Oliveira, R. E. M., Filipin, M. D. V. \& Giardini, M. H. (2015). Intervenções farmacêuticas destinadas à otimização da adesão ao tratamento medicamentoso de um paciente. Revista Eletrônica de Farmácia, 12 (2), 39-51.

Oliveros, E. et al. (2019). Hypertension in older adults: Assessment, management, and challenges. Clinical Cardiology, 43, 99-107.

Organização Pan Americana da Saúde - OPAS (2020). OMS revela principais causas de morte e incapacidade em todo o mundo entre 2000 e 2019. https://www.paho.org/pt/noticias/9-12-2020-oms-revela-principais-causas-morte-e-incapacidade-em-todo-mundo-entre-2000-e

Peacock, E. \& Krousel-Wood, M. (2017). Adherence to Antihypertensive Therapy. Medical Clinics of North America, 101 (1), $229-245$.

Pereira, A. S. et al. (2018). Metodologia da pesquisa científica. UFSM.

Pereira, M. C. A \& Santos, L. F. S. (2020). Envelhecimento saudável: relação entre hipertensão arterial sistêmica e principais fatores de riscos modificáveis. Revista Ciência Plural, 6 (1), 74-91. 
Research, Society and Development, v. 10, n. 8, e9910816939, 2021

(CC BY 4.0) | ISSN 2525-3409 | DOI: http://dx.doi.org/10.33448/rsd-v10i8.16939

Reis, W. C. T. et al. (2018). Impact of pharmacist consultation on polypharmacy patients with high cardiovascular risk. Revista Brasileira de Farmácia Hospitalar e Serviços de Saúde, 9 (2), 1-5.

Rill, J. W. G. (2016). Polifarmácia em idosos: detenção de casos no PSF Maria Oliveira de Castro do município de Aguanil/ Minas Gerais [Trabalho de Conclusão de Curso]. Minas Gerais: Universidade Federal de Minas Gerais.

Santos, A. L. S. (2018). A importância do educador físico no processo de acompanhamento e favorecimento da qualidade de vida da pessoa idosa [Trabalho de Conclusão de Curso]. Teresina: Faculdade do Médio Parnaíba.

Secretaria de Ciências, Tecnologias e Insumos Estratégicos (2014). Departamento de Assistência Farmacêutica e Insumos Estratégicos. Cuidado Farmacêutico na Atenção básica. Capacitação para implantação dos serviços de clínica farmacêutica. http://bvsms.saude.gov.br/bvs/publicacoes/cuidado_farmaceutico_atencao_basica_saude_2.pdf

Silva, M. V. F. et al. (2013). Antiagregantes plaquetários na prevenção primária e secundária de eventos aterotrombóticos. Arquivos Brasileiros de Cardiologia, 100(6).

Sociedade Brasileira de Cardiologia (2017). Atualização da Diretriz Brasileira de Dislipidemias e Prevenção da Aterosclerose. Arquivo Brasileiro de Cardiologia, 109 (2 Supl. 1).

Sousa, N. P. et al. (2016). Estratificação de risco cardiovascular na atenção primária segundo escore de Framingham. Tempus, Actas de Saúde Coletiva, 10 (1), 157-168.

Stefanello, F. P. S., Pasqualotti, A. \& Pichle, N. A. (2020). Análise do consumo de alimentos fontes de ômega 3 por participantes de grupos de convivências. Revista Brasileira de Geriatria e Gerontologia, 22 (6), 1-9.

Trautwein, E. A \& Mckay, S. (2020). The Role of Specific Components of a Plant-Based Diet in Management of Dyslipidemia and the Impact on Cardiovascular Risk. Nutrients, 12 (9), 1-21.

Weaver, C. M. et al. (2015). Calcium plus vitamin D supplementation and risk of fractures: an updated meta-analysis from the National Osteoporosis Foundation. Osteoporosis international, 27 (1), 367-76. 